\title{
Semisynthesis and in vitro anticancer activities of andrographolide analogues
}

\begin{abstract}
The plant Andrographis paniculata found throughout Southeast Asia contains Andrographolide 1, a diterpenoid lactone, which has antitumour activities against in vitro and in vivo breast cancer models. In the present study, we report on the synthesis of andrographolide derivatives, 3,19-isopropylideneandrographolide (2), 14-acetyl-3,19isopropylideneandrographolide (3) and 14-acetylandrographolide (4), and their in vitro antitumour activities against a 2-cell line panel consisting of MCF-7 (breast cancer cell line) and HCT-116 (colon cancer cell line). Compounds 2 and 4 were also screened at the US National Cancer Institute (NCI) for their activities against a panel of 60 human cancer cell lines derived from nine cancer types. Compound 2 was found to be selective towards leukaemia and colon cancer cells, and compound 4 was selective towards leukaemia, ovarian and renal cancer cells at all the dose-response parameters. Compounds 2 and 4 showed nonspecific phase of the cell cycle arrest in MCF-7 cells treated at different intervals with different concentrations. NCI's COMPARE and SOM mechanistic analyses indicated that the anticancer activities of these new class of compounds were not similar to that of standard anticancer agents, suggesting novel mechanism(s) of action.
\end{abstract}

Keyword: Andrographis paniculata, Acanthaceae, Andrographolide, Semisynthesis, Anticancer, Cell cycle 